PROCEEDINGS OF THE

AMERICAN MATHEMATICAL SOCIETY

Volume 136, Number 9, September 2008, Pages 3363-3373

S 0002-9939(08)09343-X

Article electronically published on May 6, 2008

\title{
HOMOGENEOUS AND INHOMOGENEOUS MANIFOLDS
}

\author{
PAUL GARTSIDE, DAVID GAULD, AND SINA GREENWOOD
}

(Communicated by Alexander N. Dranishnikov)

\begin{abstract}
All metaLindelöf, and most countably paracompact, homogeneous manifolds are Hausdorff. Metacompact manifolds are never rigid. Every countable group can be realized as the group of autohomeomorphisms of a Lindelöf manifold. There is a rigid foliation of the plane.
\end{abstract}

\section{INTRODUCTION}

Inspired by a recent paper, Manifolds: Hausdorffness versus homogeneity by Baillif and Gabard 1, we investigate the topology of non-Hausdorff manifolds and give applications to foliations. By a manifold we mean a connected space which is locally homeomorphic to $\mathbb{R}^{n}$ for some $n$. Manifolds are necessarily $T_{1}$, but not guaranteed to be Hausdorff. Non-Hausdorff manifolds arise naturally as quotients of Hausdorff manifolds: for example as the leaf space of a foliation [5, 6, 7, as reduced twistor spaces in relativity theory [10, and as models of space-time in 'many-worlds' interpretations of quantum mechanics [8].

It is easy to prove that Hausdorff manifolds are always homogeneous, but this is not necessarily true of $T_{1}$ manifolds. For example, take $\mathbb{R} \times\{0,1\}$ and identify $(x, 0)$ with $(x, 1)$ for all $x$ except $x=0$. The resulting space, commonly called the 'split origin' space, is a non-homogeneous Lindelöf one manifold. In [1] two examples of homogeneous non-Hausdorff manifolds are presented, but it is also shown that a Lindelöf homogeneous manifold is always Hausdorff (and hence metrizable). One is naturally led to consider under which circumstances homogeneous manifolds are Hausdorff, particularly covering properties weaker than 'Lindelöf'. Here we prove that every metaLindelöf homogeneous manifold is Hausdorff (indeed metrizable) and that most countably paracompact homogeneous manifolds are Hausdorff - but not necessarily metrizable. Further one wants to know how 'badly' non-Hausdorff manifolds can fail to be homogeneous. A space whose sole autohomeomorphism is the identity map is said to be 'rigid'. Thus rigidity and homogeneity are polar opposites. We show that metacompact manifolds are never rigid, but there is a rigid Lindelöf one manifold. Using this space we further show that every countable group can be realized as the group of autohomeomorphisms of a Lindelöf one manifold.

Received by the editors May 22, 2007, and, in revised form, August 8, 2007.

2000 Mathematics Subject Classification. Primary 54D10, 54D20, 57R30, 57N05, 57S05.

Key words and phrases. Manifold, not Hausdorff, homogeneous, rigid, foliations.

This work was supported in part by the Marsden Fund Council from government funding, administered by the Royal Society of New Zealand. 
Finally we give applications of our results to foliations of the plane. From our rigid manifold we describe an explicit construction of a rigid foliation of the plane.

\section{Covering PROPERTIES OF NON-HAUSDORFF MANIFOldS}

Recall that a topological space is 'Lindelöf' if every open cover has a countable subcover. Further, a space is 'paracompact' if every open cover has a locally finite open refinement, and is 'metacompact' if every open cover has a point-finite open refinement. Finally, a space is 'metaLindelöf' if every open cover has a pointcountable open refinement.

It is easy to see, because manifolds are locally second countable, that 'second countable' and 'Lindelöf' coincide in manifolds.

Paracompact vs. metacompact vs. MetaLindelöf in manifolds.

Lemma 2.1. Lindelöf and metaLindelöf are equivalent in manifolds.

Proof. We show that a metaLindelöf manifold is Lindelöf. We need two facts. First, every point-countable open cover of a locally separable space has a starcountable open refinement (each element of the cover meets only countably many other members of the cover). Second, any star-countable collection $\mathcal{V}$ can be written $\mathcal{V}=\bigcup_{\lambda \in \Lambda} \mathcal{V}_{\lambda}$ where each $\mathcal{V}_{\lambda}$ is countable and $\left(\bigcup \mathcal{V}_{\lambda}\right) \cap\left(\bigcup \mathcal{V}_{\lambda^{\prime}}\right)=\emptyset$ whenever $\lambda \neq \lambda^{\prime}$.

Thus given any open cover $\mathcal{U}$ of a metaLindelöf manifold $M$, let $\mathcal{V}$ be a pointcountable open refinement and $\mathcal{W}$ a star-countable open refinement of $\mathcal{V}$. Partition $\mathcal{W}$ as above. Since $M$ is connected there can only be one element of $\Lambda$, and so $\mathcal{W}$ is countable and hence $\mathcal{U}$ has a countable subcover.

Since 'paracompact' implies 'metacompact' which implies 'metaLindelöf':

Corollary 2.2. Metacompact (so paracompact) manifolds are second countable.

Example 2.3. There is a second countable manifold which is not metacompact.

Proof. Let $M=\mathbb{R} \cup(\mathbb{Q} \times \mathbb{N} \times[0, \infty))$ with the topology in which points of $\mathbb{R}$ and $\{(q, n)\} \times(0, \infty)$ have their standard neighbourhoods (here $q \in \mathbb{Q}$ and $n \in \mathbb{N}$ ), and a basic neighbourhood of $(q, n, 0)$ is $B(q, n, \epsilon)=(q-\epsilon, q) \cup\{(q, n)\} \times[0, \epsilon)$, where $\epsilon>0$. Then $M$ is a one dimensional second countable manifold.

We show that $M$ is not metacompact. Let $\mathcal{U}=\{\mathbb{R}\} \cup\{\{(q, n)\} \times(0, \infty): q \in$ $\mathbb{Q}, n \in \mathbb{N}\} \cup\{B(q, n, 1 / n): q \in \mathbb{Q}, n \in \mathbb{N}\}$, an open cover of $M$. Suppose $\mathcal{V}$ is any open refinement. For each $(q, n)$ pick $\epsilon_{q, n}>0$ such that $B\left(q, n, \epsilon_{q, n}\right)$ is contained in some element of $\mathcal{V}$. Note that $\epsilon_{q, n}<1 / n$.

Let $W_{n}=\bigcup_{q \in \mathbb{Q}}\left(q-\epsilon_{q, n}, q\right)$, which is an open dense subset of the reals, and hence there is an $x \in \bigcap_{n} W_{n}$. So for each $n$ there is $q_{n} \in \mathbb{Q}$ with $x \in\left(q_{n}-\epsilon_{q_{n}, n}, q_{n}\right)$.

If infinitely many of the elements $q_{n}$ are different, then we see that the point $x$ of $M$ is in infinitely many (distinct) elements of $\mathcal{V}$. On the other hand, if infinitely many of the elements $q_{n}$ are all equal (say to $q$ ), since $\epsilon_{q, n} \rightarrow 0$, we see that $x$ is again in infinitely many elements of $\mathcal{V}$. In either case $\mathcal{V}$ is not point-finite.

Example 2.4. There is a metacompact manifold which is not paracompact.

Proof. Let $X=\mathbb{R} \times \mathbb{N}$. For each $x<0$ in the real line, identify $(x, n)$ with $(x, m)$ for all $n, m \in \mathbb{N}$, and call it $x$. Let $M$ be the resulting quotient space. Then $M$ is a one dimensional second countable manifold which can be described as the line with a countable infinity of branches at the origin'. 
Take any open cover $\mathcal{U}$ of $M$. Since $(-\infty, 0) \cup \bigcup_{n}(0, \infty) \times\{n\}$ is metacompact, there is a partial point-finite open refinement $\mathcal{V}^{\prime}$ of $\mathcal{U}$ covering all points except the points $(0, n)$. For each $n$ pick $m_{n} \geq n$ so that $V_{n}=\left(-1 / m_{n}, 0\right) \cup\left[0,1 / m_{n}\right) \times\{n\}$ is contained in some element of $\mathcal{U}$. Now $\mathcal{V}=\mathcal{V}^{\prime} \cup\left\{V_{n}: n \in \mathbb{N}\right\}$ is a point-finite open refinement of $\mathcal{U}$, as required for $M$ to be metacompact.

Since every neighbourhood of $(0, m)$ meets every neighbourhood of $(0, n)$, it is clear $M$ is not paracompact.

\section{When homogeneous manifolds are HausdorfF}

We start with a general discussion of 'points of Hausdorffness' and 'points of regularity' in manifolds. This will be applied below and in the next section.

For any subset $A$ of a space $X$, let $R(A)$ consist of all points of $A$ with a neighbourhood base in $X$ of closed sets. Obviously $X$ is regular if and only if $R(X)=X$. Define $H(A)$ to be all points of $X$ which can be separated by disjoint open sets from every other point of $A$, and $N H(A)=X \backslash H(A)$. Write $N H(x)$ for $N H(\{x\})$, so $N H(x)$ consists of all points that cannot be Hausdorff separated from $x$.

In the case of a manifold we clearly have:

Lemma 3.1. For an m-manifold $M, R(M)=$ all points with a closed neighbourhood homeomorphic to a closed ball in $\mathbb{R}^{m}$. Hence $R(M)$ is open.

Lemma 3.2. In a manifold $M$, the set $N H(x)$ is closed for every point $x$ of $M$.

Lemma 3.3. Let $M$ be a manifold. Then $R(M)=\emptyset$ if and only if $N H(M)$ is dense. Also, $R(M)$ is not dense if and only if $N H(M)$ is somewhere dense (dense in some non-empty open set).

Proof. If $R(M)$ is non-empty, then it contains a (non-empty) open set. This set clearly cannot contain any non-Hausdorff points, so $N H(M)$ is not dense.

If $N H(M)$ is not dense, then $H(M)$ contains a point with a compact neighbourhood homeomorphic to a Euclidean closed ball, $K$. Every point of $M \backslash K$ can be separated from $K$ by an open set, so $K$ is closed in $M$, and Lemma 3.1 applies.

The second claim follows similarly.

Lemma 3.4. If $R(M) \neq \emptyset$, where $M$ is a homogeneous manifold, then $M$ is regular $(R(M)=M)$ (and Hausdorff, of course).

Corollary 3.5. MetaLindelöf homogeneous manifolds are Hausdorff (indeed metrizable).

Proof. This follows from Lemma 2.1 and [1, Theorem 1.1].

A space is 'countably compact' ('countably paracompact') if every countable open cover has a finite subcover (a locally finite open refinement). Countably compact spaces are countably paracompact. The long line is a Hausdorff manifold which is countably compact but not Lindelöf. We now show that in most circumstances, countably paracompact homogeneous manifolds are Hausdorff.

Let $X$ be a space, and $A, B$ any subsets of $X$. Define $\rho(A, B)$ (the 'reflection' of $A$ in $B)$ to be $\rho(A, B)=\{x \in B: N H(x) \cap A \neq \emptyset\}$.

Lemma 3.6. Let $M$ be a manifold. If $U$ and $V$ are Euclidean open sets in $M$, then $\rho(V, U)$ is nowhere dense in $U$. 
Proof. Let $I$ be the interior of the closure of $\rho(V, U)$ in the subspace $U$, and suppose that $I \neq \emptyset$. Note that $\rho(V, U)$ is dense in $I$; take any $p \in \rho(V, U) \cap I$ and any $p^{\prime} \in N H(p) \cap V$. Each neighbourhood of $p^{\prime}$ meets each neighbourhood of $p$, so $V \cap I \neq \emptyset$. Now $V \cap I$ is open in $I$ and $\rho(V, U)$ is dense in $I$, hence $\rho(V, U) \cap V \cap I \neq \emptyset$, a contradiction as $V \cap \rho(V, U)=\emptyset$ since $V$ is Euclidean open.

Theorem 3.7. Let $M$ be a countably paracompact homogeneous manifold. If for some $x \in M$ either (i) $N H(x)$ is not countably compact or (ii) $N H(x)$ is Lindelöf, then $M$ is Hausdorff.

Proof. Let $M$ be a homogeneous non-Hausdorff manifold. We show that if either (i) or (ii) hold, then the manifold is not countably paracompact.

(i): First suppose $x$ is in $M$ and $N H(x)$ is not countably compact; equivalently $N H(x)$ contains an infinite closed discrete subspace, $C=\left\{x_{n}\right\}_{n \in \mathbb{N}}$. Since $N H(x)$ is closed in $M, C$ is closed discrete in $M$. For each $x_{n}$ pick an open Euclidean neighbourhood, $T_{n}$, witnessing closed discreteness. Now $\mathcal{U}=\{M \backslash C\} \cup\left\{T_{n}: n \in \mathbb{N}\right\}$ is a countable open cover of $M$, no open refinement of which is locally finite at $x$.

(ii): Now suppose that $x$ is in $M$ and $N H(x)$ is Lindelöf. By homogeneity and non-Hausdorffness of $M$, every $N H(y)$ is (Lindelöf and) non-empty. Fix Euclidean open $U$ containing $x$. We claim there are sequences $\left(x_{n}\right)_{n}$ and $\left(x_{n}^{\prime}\right)_{n}$ where: each $x_{n}$ is in $U$ and $x_{n} \rightarrow x$; and $x_{n}^{\prime} \in N H\left(x_{n}\right)$, for each $n$; but $C=\left\{x_{n}^{\prime}: n \in \mathbb{N}\right\}$ is closed discrete. Let $T_{n}$ witness closed discreteness for $x_{n}$. Then $\mathcal{U}=\{M \backslash C\} \cup\left\{T_{n}: n \in \mathbb{N}\right\}$ is a countable open cover of $M$, no open refinement of which is locally finite at $x$.

To establish the claim, note that if $\left(x_{n}\right)$ is a sequence on $U$ converging to $x$, and $x_{n}^{\prime}$ is in $N H\left(x_{n}\right)$ for each $n$, then $\left(x_{n}^{\prime}\right)$ can converge only to elements of $N H(x)$.

For each $y \in N H(x)$ pick a Euclidean open $V_{y}$ containing $y$. As $N H(x)$ is Lindelöf, we can find a countable subcollection $V_{y_{1}}, V_{y_{2}}, \ldots$ covering $N H(x)$. By Lemma 3.6, $\rho\left(V_{y_{i}}, U\right)$ is nowhere dense in $U$. So $U \backslash \bigcup_{i=1}^{\infty} \rho\left(V_{y_{i}}, U\right)$ is dense in $U$. Thus we can pick a sequence $\left(x_{n}\right)_{n}$ from this set converging to $x$, and pick $x_{n}^{\prime}$ in $N H\left(x_{n}\right)$ for each $n$. No $x_{n}^{\prime}$ is in any $V_{y_{i}}$; otherwise $x_{n}$ is in $\rho\left(V_{y_{i}}, U\right)$. Hence they cannot converge to anything in $N H(x)$ (or, as observed above, anything else).

\section{Rigidity AND NON-RIGIDITY}

We start this section by giving conditions under which a manifold is not rigid. Then we construct a variety of rigid manifolds, and manifolds with specified autohomeomorphism or homeotopy group.

\section{Conditions for non-rigidity.}

Lemma 4.1. If $M$ is a manifold and $R(M) \neq \emptyset$, then $M$ is not rigid; indeed, the autohomeomorphism group of $M$ contains many uncountable free subgroups.

Proof. To see this fix an open set $U$ in $M$ homeomorphic to some $\mathbb{R}^{m}$ with closure homeomorphic to the closed ball in $\mathbb{R}^{m}$. Then any non-identity homeomorphism of the closure of $U$ which is the identity on the boundary of $U$ can be extended to a non-trivial homeomorphism of $M$ which is the identity everywhere outside $U$.

A minor modification of the argument in [2] shows that in fact the group of all autohomeomorphisms of the closed ball in $\mathbb{R}^{m}$ fixing the boundary is 'almost free' (almost all, in the sense of Baire category, $n$-tuples of autohomeomorphisms freely generate a free subgroup). Hence, see [4], almost all (again in the sense of Baire 
category) uncountable compact subsets of the autohomeomorphism group freely generate a free subgroup.

Theorem 4.2. Every metacompact manifold $M$ has (open) dense $R(M)$, and hence is far from being rigid.

Proof. The 'and hence' part follows because as $R(M)$ is open and dense it is nonempty and Lemma 4.1 applies.

Fix a metacompact manifold $M$ and suppose that $R(M)$ is not dense. By Lemma $3.3 N H(M)$ is dense in some open subset $U$ of $M$ homeomorphic to $\mathbb{R}^{m}$.

Let $\mathcal{U}$ be an open cover of $M$ by sets homeomorphic to some $\mathbb{R}^{m}$. Let $\mathcal{V}$ be any open refinement of $\mathcal{U}$. We show $\mathcal{V}$ is not point-finite.

Let $D=\left\{x_{n}\right\}_{n \in \mathbb{N}}$ be a dense set of non-Hausdorff points in $U$. Let $D^{\prime}=\left\{x_{n}^{\prime}\right\}_{n \in \mathbb{N}}$ be such that $x_{n}^{\prime}$ cannot be Hausdorff separated from $x_{n}$. Note that each $x_{n}^{\prime}$ is not in $U$, but is in $\bar{U}$. For each $n$, pick $V_{n} \in \mathcal{V}$ so that $x_{n}^{\prime} \in V_{n}$. Let $W_{n}=V_{n} \cap U$. Then $\left\{W_{n}\right\}_{n}$ is a collection of open sets in $\mathbb{R}^{m}$ such that $x_{n} \in \overline{W_{n}} \backslash W_{n}$ for each $n$.

We show this latter collection is not point-finite: set $n_{1}=1$, select a non-empty open $S_{1}$ whose closure in $U$ lies in $W_{n_{1}}$, and inductively pick $n_{i}$ so that $x_{n_{i}} \in S_{i-1}$, and select a non-empty open $S_{i}$ whose closure in $U$ lies in $S_{i-1} \cap W_{n_{i}} \subseteq \bigcap_{j \leq i} W_{n_{j}}$. Then there is a point $z \in \bigcap_{i \in \mathbb{N}} S_{i}$, and this point is in infinitely many of the sets $W_{n}$ (namely $W_{n_{i}}$ for each $i$ ).

If every Lindelöf manifold had $R(M)$ non-empty, this would give an alternative proof that Lindelöf homogeneous manifolds are Hausdorff. But this is not the case.

Example 4.3. There is a second countable manifold with empty $R(M)$. The given example is not rigid.

Proof. The manifold is called 'La plume composée' in [6]. We start by describing the 'plume': the underlying set is $P=\mathbb{R} \cup(\mathbb{Q} \times[0, \infty))$, points in the 'shaft' $(0, \infty)$ have their usual neighbourhoods, a basic neighbourhood of $(q, 0)$ is $B(q, 0, \epsilon)=$ $\{q\} \times[0, \epsilon) \cup(q, q+\epsilon)$, and points on the 'barb' $\{q\} \times(0, \infty)$ have their usual neighbourhoods (in the 'barb').

Now we can get the 'plume double' by replacing each 'barb' by a 'plume' and iterate countably many times. This gives a second countable one dimensional manifold $M$, the 'plume composée'. Note that this manifold is far from being rigid.

The key property of this example is that the set of non-Hausdorff points is dense. Hence Lemma 3.3 applies.

\section{Rigid manifolds.}

Example 4.4. There is a second countable rigid 1-manifold.

Proof. For each $n \geq 1$ let $M(n)=((-1,0) \cup([0,1) \times\{1, \ldots, n\})) \times\{n\}$ with topology where points with a real part non-zero have their usual Euclidean neighbourhoods, and a basic neighbourhood of $(0, i)$ is $B(0, i, \epsilon)=(-\epsilon, 0) \cup([0, \epsilon) \times\{i\})$. Then $M(n)$ is a second countable one dimensional manifold with an order inherited from the order on the real parts. Call points with a real part $<0$ the 'left part' of $M(n)$, call points with a real part $>0$ the 'right part' of $M(n)$, and call the points $(0, i)$ the 'branch points'. The branch points are the non-Hausdorff points of $M(n)$.

Let $X=(0,1) \cup \bigcup_{n \geq 1} M(n)$. Fix a countable dense set $D$ of the union of $(0,1)$ and the right parts of the $M(n)$. Enumerate $D=\left\{x_{m}\right\}_{m \geq 1}$. For each $m \geq 1$ 
fix a neighbourhood $U_{m}$ of $x_{m}$ and a homeomorphism $h_{m}:(-1,1) \rightarrow U_{m}$ so that $h_{m}(0)=x_{m}, x_{i} \notin U_{m}$ for $i<m$ and $U_{m}$ contains no branch points. We may assume that $h_{m}$ preserves the order. We think of the domain of $h_{m}$ as being the shaft of $M(m)$, i.e. as $((-1,0) \cup([0,1) \times\{1\})) \times\{m\}$. Identify the left part of $M(m)$ with $h_{m}((-1,0))$ via $h_{m}$ for each $m$. This gives a second countable 1 -manifold $M$. (If we arrange that $x_{m} \notin M(n)$ for $n \geq m$, then $M$ will have no 'loops', and so is orientable.)

We prove that $M$ is rigid. Note first that the non-Hausdorff points, $N H(M)$, are the branch points and the points of $D$, and are dense. Take any autohomeomorphism $h$ of $M$. Then $h$ carries (non-) Hausdorff points to (non-) Hausdorff points. Thus if $h$ is the identity on $N H(M)$ (which is dense), by Hausdorffness of all other points of $M, h$ is forced to be the identity on the whole of $M$.

It remains to show that $h$ is indeed the identity on $N H(M)$. To do this we associate with each point of $N H(M)$ an object which is invariant under homeomorphisms and so that distinct points of $N H(M)$ receive different objects.

The relation $\sim$ on $N H(M)$ given by $x \sim x^{\prime}$ if and only if $x$ and $x^{\prime}$ cannot be Hausdorff separated is, for this particular manifold, an equivalence relation. So $N H(M)$ is partitioned into equivalence classes $N_{m}$, for $m \geq 1$, where $N_{m}$ consists of $x_{m}$ and the branch points of $M(m)$. Note that the definition of $N_{m}$ is purely topological: it is the unique equivalence class of $\sim$ with precisely $m+1$ elements.

Let $\sigma(x)=\left\{\left\{\left|N_{m}\right|: N_{m} \cap U \neq \emptyset\right\}: U\right.$ is an open neighbourhood of $\left.x\right\}$ for $x \in N H(M)$. Then it is easy to check that $\sigma(x)=\sigma(h(x))$ (so $\sigma$ is invariant) and $\sigma(x) \neq \sigma\left(x^{\prime}\right)$ for any distinct $x, x^{\prime} \in N H(M)$ — as required.

Example 4.5. There are rigid manifolds of arbitrarily large cardinality.

Proof. It is known that for every cardinal $\kappa$, there is a cardinal $\lambda \geq \kappa$ so that $\aleph_{\lambda}=\lambda$. Here $\aleph_{\alpha}$ denotes the $\alpha$ th infinite cardinal.

Repeat the argument for Example 4.4 with the following changes. For each $\beta \leq \lambda$ let $M\left(\aleph_{\beta}\right)=\left((-1,0) \cup\left([0,1) \times\left\{\aleph_{\beta}: \beta \leq \lambda\right\}\right)\right) \times\{\beta\}$ with obvious topology. Let $X=(0,1) \cup \bigcup_{\beta \leq \lambda} M\left(\aleph_{\beta}\right)$. Let $D$ be a dense subset of $(0,1)$ and the right parts of the $M\left(\aleph_{\beta}\right)$ such that $|D|=\aleph_{\lambda}$. Now proceed as before. The $\sim$-equivalence classes all have different cardinalities, and so the invariant $\sigma$ works as before.

Realizing groups as autohomeomorphism groups of manifolds. Let $G$ be a group. The directed graph with vertices $G$ and an edge from $g$ to $g^{\prime}$ of colour $h$ if and only if $g . h=g^{\prime}$ is called the Cayley graph of $G$, denoted $C G(G)$. An automorphism of $C G(G)$ is a permutation $\alpha$ of the vertices such that $\left(g, g^{\prime}\right)$ is an edge of $C G(G)$ if and only if $\left(\alpha(g), \alpha\left(g^{\prime}\right)\right)$ is an edge of $C G(G)$. Further, $\alpha$ is said to be colour preserving if for every edge $\left(g, g^{\prime}\right)$, the edges $\left(g, g^{\prime}\right)$ and $\left(\alpha(g), \alpha\left(g^{\prime}\right)\right)$ have the same colour. The key property of the Cayley graph is that its group of colour preserving automorphisms, Aut $(C G(G))$, is isomorphic to $G$.

Theorem 4.6. For every countable group $G$ there is a second countable one dimensional manifold $M(G)$ such that the group of autohomeomorphisms of $M(G)$ is isomorphic to $G$.

Proof. Given a countable group $G=\left\{g_{n}\right\}_{n \in \mathbb{N}}$ we describe a second countable one dimensional manifold $M(G)$ whose group of autohomeomorphisms, $\mathcal{H}(M(G)$ ), is isomorphic to $\operatorname{Aut}(C G(G)$ ) (and hence $G$ ). The rigid manifold $M$ of Example 4.4 
is the main building block, playing the roles of both vertices and edges of the Cayley graph of $G$. The terminology and notation of that example is used here.

Fix two disjoint countable subsets of $(0,1)$ in $M$, both disjoint from $D$, and label them $g_{1}, g_{2}, \ldots$ and $g_{1}^{\prime}, g_{2}^{\prime}, \ldots$ Think of the points $g_{i}$ and $g_{i}^{\prime}$ as having 'colour' $g_{i}$. An edge joining $g_{i}$ to $g_{i}^{\prime}$ will be considered to 'start' at $g_{i}$ and 'end' at $g_{i}^{\prime}$ and will inherit their colour $g_{i}$. Extend $(0,1)$ in $M$ left and right to $(-1,2)$ in the natural way to get manifold $M^{\prime}$ (so points of $(-1,2)$ have their standard neighbourhoods).

Let $\mathbf{V}=M \times G$. Think of $M \times\{g\}$ as being the vertex $g$. Let $\mathbf{E}=M^{\prime} \times(G \times G)$. Think of $M^{\prime} \times\{g\} \times\{h\}$ as being an edge (as yet unattached). Once attached (using the 'flaps' $(-1,0)$ and $(1,2))$, it will start at vertex $g$ and end at vertex $g$.h. Let $X(G)=\mathbf{V} \cup \mathbf{E}$.

For each $g \in G$ and $g_{i} \in G$ identify the left part of a small neighbourhood of the point $g_{i}$ in 'vertex' $M \times\{g\}$ with the 'left flap' $(-1,0)$ in the 'edge' $M^{\prime} \times\{g\} \times\left\{g_{i}\right\}$, and identify the right part of a small neighbourhood of the point $g_{i}^{\prime}$ in 'vertex' $M \times\left\{g . g_{i}\right\}$ with the 'right flap' $(1,2)$ in the 'edge' $M^{\prime} \times\{g\} \times\left\{g_{i}\right\}$. This gives a second countable one dimensional manifold $M(G)$.

Then $M(G)$ is as required: $\mathcal{H}(M(G))=\operatorname{Aut}(C G(G))$; see the proof of Theorem 2 from [3] for a similar argument.

Using Example 4.5 and the technique of the preceding theorem it is clear that:

Theorem 4.7. For every group $G$ there is a manifold $M$ whose autohomeomorphism group is isomorphic to $G$.

Isotopy. Let $M$ be a manifold. Let $\mathcal{I}$ be all $h \in \mathcal{H}(M)$ which are isotopic to the identity. Then $\mathcal{I}$ is a normal subgroup of $\mathcal{H}(M)$. The quotient $\mathcal{H}(M) / \mathcal{I}$ is the 'homeotopy group' of $M$.

Take any countable group $G$, and consider the manifold $M(G)$ of Theorem 4.6 whose group of autohomeomorphisms coincides with $G$. If $M(G)$ were Hausdorff, then an isotopy between any two distinct autohomeomorphisms would give a nontrivial path in $\mathcal{H}(M(G))$ - which is impossible as non-trivial paths are uncountable and $\mathcal{H}(M(G))$ is countable. Since $M(G)$ is not Hausdorff this argument cannot be applied directly. Nevertheless, given $h \neq g$ in $\mathcal{H}(M(G))$ and a point $x \in M(G)$ with $h(x) \neq g(x)$, an isotopy between $h$ and $g$ gives a non-trivial path in $M(G)$ which should only have countably many values, and this is impossible in a manifold. Thus:

Theorem 4.8. Every countable group can be realized as the homeotopy group of a second countable manifold.

\section{Applications to Foliations}

A partition $\mathcal{F}=\left\{L_{\alpha}: \alpha \in A\right\}$ of an $n$-manifold $M$ by arcwise connected subsets is a $k$-dimensional foliation of $M$, where $0 \leq k \leq n$, provided:

- for each $p \in M$ there is a chart $(U, \varphi)$ on $M$ about $p$ such that for each $\alpha \in A$ if $L_{\alpha} \cap U \neq \emptyset$, then each arcwise component of $\varphi\left(L_{\alpha} \cap U\right)$ is of the form $\left\{\left(x_{1}, \ldots, x_{n}\right) \in \varphi(U): x_{k+1}=c_{k+1}, \ldots, x_{n}=c_{n}\right\}$, where $c_{k+1}, \ldots, c_{n}$ are constants determined by the component.

Each set $L_{\alpha}$ is a leaf of the foliation. The set of leaves is the leaf space; it carries the obvious quotient topology from $M$. By an oriented foliation we mean that 
each leaf has been given an orientation in such a way that neighbouring leaves have compatible orientations. See [9] for an introduction to foliations.

We are interested here only in foliations where $n=2$, usually $M=\mathbb{R}^{2}$, and $k=1$. In this case every foliation is orientable, and the leaf space is a simply connected, orientable, Lindelöf one manifold (not necessarily Hausdorff). Haefliger and Reeb showed that plane foliations are nearly classified by their leaf spaces. A complete classification requires some additional structure on the non-Hausdorff points of the leaf space.

Let $M$ be a simply connected, orientable, Lindelöf one manifold with oriented atlas $\mathcal{A}$. Define equivalence relations $\sim^{+}$and $\sim^{-}$on $N H(M)$ as follows. Let $x_{1}$ and $x_{2}$ be two points of $M$ which are not Hausdorff separated. Pick $\left(U_{1}, \phi_{1}\right),\left(U_{2}, \phi_{2}\right) \in \mathcal{A}$ such that $x_{i} \in U_{i}$, and $\phi_{i}:(-1,1) \rightarrow U_{i}$ is an orientation preserving homeomorphism, for $i=1,2$. Set $x_{1} \sim^{-} x_{2}$ if ' $U_{1}$ and $U_{2}$ meet on the left': $\phi_{1}[(-1,0)] \cap$ $\phi_{2}[(-1,0)] \neq \emptyset$. Set $x_{1} \sim^{+} x_{2}$ if ' $U_{1}$ and $U_{2}$ meet on the right': $\phi_{1}[(0,1)] \cap$ $\phi_{2}[(0,1)] \neq \emptyset$. These equivalence relations have countable equivalence classes.

An 'order structure' on a simply connected, orientable, Lindelöf one manifold is an explicit total order structure on the $\sim^{+}$and $\sim^{-}$equivalence classes.

Theorem 5.1 (Haefliger \& Reeb, 6]). There is a bijective correspondence between oriented foliations of the plane and Lindelöf, one dimensional, orientable, simply connected manifolds with an order structure.

It is shown in [6] that a one manifold is simply connected if and only if every point is a cut point (remove that point and the space is disconnected). This makes identifying simply connected one manifolds very straightforward.

Thus, Examples 2.3 and 2.4 are both second countable, orientable, simply connected 1-manifolds, so there are foliations of the plane whose leaf spaces are (1) not metacompact and (2) metacompact but not paracompact, respectively.

An automorphism of an oriented foliation $\mathcal{F}$ is an orientation preserving autohomeomorphism of the plane taking leaves to leaves. Denote the group of all automorphisms of $\mathcal{F}$ by $\operatorname{Aut}(\mathcal{F})$. Call an automorphism 'inner' if it carries each leaf to itself. Let $\operatorname{Inn}(\mathcal{F})$ be the collection of all inner automorphisms of $\mathcal{F}$. Let $\operatorname{Out}(\mathcal{F})=\operatorname{Aut}(\mathcal{F}) / \operatorname{Inn}(\mathcal{F})$. Every automorphism of a foliation $\mathcal{F}$ induces an autohomeomorphism of the leaf space. Two automorphisms of a foliation yield the same autohomeomorphism of the leaf space if and only if they differ only by an inner automorphism. The correspondence theorem implies that any autohomeomorphism of a leaf space can be lifted to an automorphism of the foliation provided it respects the order structure.

Lemma 5.2. Let $\mathcal{F}$ be a foliation of the plane. Then $\operatorname{Inn}(\mathcal{F})$ contains an uncountable free subgroup. In particular, the full automorphism group is never trivial.

Proof. The group of all orientation preserving autohomeomorphisms of $(-1,1)$ contains many uncountable free subgroups because it is almost free [4. We show it embeds inside $\operatorname{Inn}(\mathcal{F})$.

Fix any element $U$ in a chart for $\mathcal{F}$ with $U$ homeomorphic to $(-2,2)^{2}$ and the leaves corresponding to horizontal lines. Take any orientation preserving autohomeomorphism $h$ of $(-1,1)$. Then $h$ can be extended to an inner automorphism of $\mathcal{F}$ as follows. First make it the identity outside $U$. Now identify $U$ with $(-2,2)^{2}$, and declare the extension to be the identity outside $V=\{(x, y) \in U:|x|+|y|<1\}$, and to be $(h(x /(1-|y|)), y)$ for $(x, y)$ in $V$. 
A foliation is said to be 'rigid' if all its automorphisms are inner, in other words Out is trivial, or equivalently its leaf space is a rigid manifold.

Example 5.3. There is a foliation of the plane which is rigid.

Proof. The rigid manifold of Example 4.4 is a second countable one manifold, which can be made to be orientable, as explained in the construction, and in this case it is clear every point is a cut point. So the claim follows as explained above.

It seems interesting to provide an explicit construction of the rigid foliation whose leaf space is homeomorphic to the rigid manifold of Example 4.4. particularly because the procedure for generating a foliation from an oriented, simply connected, second countable one manifold is not detailed in [6].

The 'ingredients' of the rigid manifold are: a copy of $(0,1)$; for each $n \geq 1, n$ copies of $[0,1)$; and a 'nice' enumeration of a countable dense set of all the open intervals $(0,1)$. The construction glues the ' $n$ copies of $[0,1)$ ' to the $n$th element of the dense set. Denote by $M_{n}$ the manifold obtained at the $n$th step of this process.

Analogously, the rigid foliation is made from: a copy of $(0,1)^{2}$ foliated by the vertical lines $(0)$; and for each $n \geq 1, n$ copies of $[0,1) \times(0,1)$ foliated by vertical lines (n); and a nice enumeration of a dense countable family of leaves. We think of each $[0,1) \times(0,1)$ as being 'buckled over', see the diagram. The 'nice' enumeration uses the natural enumeration of the dyadic rationals between 0 and 1, namely: $1 / 2,1 / 4,3 / 4,1 / 8,3 / 8,5 / 8,7 / 8,1 / 16, \ldots$ In fact the dense leaves are enumerated: $(1 / 2,0) ;(1 / 4,0),(1 / 2,1,1) ;(3 / 4,0),(1 / 4,1,1),(1 / 2,2,1),(1 / 2,2,2)$; $(1 / 8,0),(3 / 4,1,1),(1 / 4,2,1),(1 / 4,2,2),(1 / 2,3,1),(1 / 2,3,2),(1 / 2,3,3) ; \ldots$. Here $(x, 0)$ stands for the leaf $\{x\} \times(0,1)$ in $(0,1)^{2}$, and $(x, n, m)$ stands for the leaf $\{x\} \times(0,1)$ in the $m$ th copy of $[0,1) \times(0,1)$ of the $n$th group.

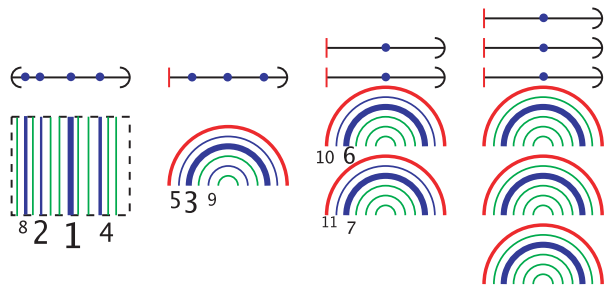

The construction starts with $\mathcal{F}_{0}$ which is just the copy of $(0,1)^{2}$ foliated by the vertical lines. At stage $n$ of the construction the $n$ 'buckles' are inserted into a tubular neighbourhood of the $n$th dense leaf, as in the diagram for two buckles.

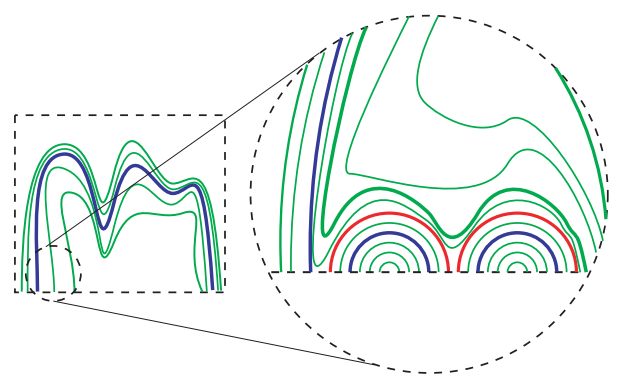

This gives a foliation $\mathcal{F}_{n}$ whose leaf space is $M_{n}$. (The diagram below shows the case $n=9$.) In the limit we get our rigid foliation. 


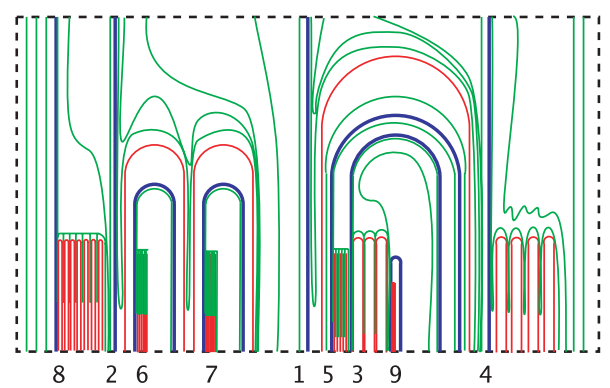

Theorem 5.4. Every finite group can be realized as the outer automorphisms of a foliation of an open (metrizable) surface.

Proof. Given a finite group $G$, construct the manifold $M(G)$ of Theorem 4.6. This has the property that its autohomeomorphism group is isomorphic to $G$. Since $M(G)$ is constructed from finitely many copies of the small rigid manifold (Example 4.4) it is easy to see that $M(G)$ is a non-Hausdorff one manifold with a finitely generated fundamental group. Further, because of the property of the rigid manifold, $M$, that the invariant $\sigma(x)$ is different for every point of $M$, any autohomeomorphism of $M(G)$ must respect any order structure placed upon it.

Haefliger ([5], page 8) has shown: for every orientable, non-Hausdorff, Lindelöf 1-manifold with a finitely generated fundamental group, $M$, there is an orientable foliation $\mathcal{F}$ by lines on a (metrizable) surface $S$, such that the leaf space of $\mathcal{F}$ is $M$.

Now the surface $S$ and foliation $\mathcal{F}$ for $M(G)$ given by Haefliger's result are as required.

Question 5.5. Which finite groups can be represented as the group of outer automorphisms of a foliation of the plane? What about the cyclic group of order $3 ?$

\section{REFERENCES}

1. M. Baillif and A. Gabard, Manifolds: Hausdorffness versus homogeneity, Proc. Amer. Math. Soc. 136 (2008), 1105-1111. MR2361887

2. A. Blass and J. Kister, Free subgroups of the homeomorphism group of the reals, Topology Appl. 24 (1986), 243-252. MR872496 (88f:20039)

3. P. Gartside and A. Glyn, Autohomeomorphism groups, Topology Appl. 129 (2003), 103-110. MR.1961392 (2004g:54044)

4. P. Gartside and R. Knight, Ubiquity of free subgroups, Bull. Lond. Math. Soc. 35 (2003), 624-634. MR.1989491 (2004e:22002)

5. A. Haefliger, Sur les feuilletages des variétés de dimension $n$ par des feuilles fermées de dimension $n-1$, Colloque de Topologie de Strasbourg, 1955.

6. A. Haefliger and G. Reeb, Variétés (non séparées) à une dimension et structures feuilletées du plan, Enseignement Math. (2) 3 (1957), 107-125. MR0089412 (19:671c)

7. S. Harris and R. Low, Causal monotonicity, omniscient foliations and the shape of space, Classical Quantum Gravity 18 (2001), 27-43. MR.1805136 (2001i:53115)

8. S. Hawking and G. Ellis, The Large Scale Structure of Space-Time, Cambridge University Press, London-New York, 1973. MR0424186 (54:12154) 
9. I. Tamura, Topology of Foliations: An Introduction, Transl. Math. Monogr., vol. 97, Amer. Math. Soc., Providence, RI, 1992. MR.1151624 (93c:57021)

10. N. Woodhouse and L. Mason, The Geroch group and non-Hausdorff twistor spaces, Nonlinearity 1 (1988), 73-114. MR928949 (89j:83034)

Department of Mathematics, University of Pittsburgh, Pittsburgh, Pennsylvania 15260

E-mail address: gartside@math.pitt.edu

Department of Mathematics, University of Auckland, Auckland, New Zealand

E-mail address: d.gauld@auckland.ac.nz

Department of Mathematics, University of Auckland, Auckland, New Zealand

E-mail address: s.greenwood@auckland.ac.nz 